\title{
Effectiveness of Prenatal Tetanus, Diphtheria, Acellular Pertussis Vaccination in the Prevention of Infant Pertussis in the U.S.
}

\author{
Sylvia Becker-Dreps, MD, MPH, ${ }^{1,2}$ Anne M. Butler, PhD, ${ }^{2,3,4}$ Leah J. McGrath, PhD, ${ }^{5}$ \\ Kim A. Boggess, MD, ${ }^{6}$ David J. Weber, MD, MPH, ${ }^{7}$ Dongmei Li, MS, ${ }^{2}$ \\ Michael G. Hudgens, $\mathrm{PhD}{ }^{8}$ J. Bradley Layton, $\mathrm{PhD}^{2,9}$
}

This activity is available for CME credit. See page A3 for information.

\begin{abstract}
Introduction: It is recommended that all pregnant women in the U.S. receive tetanus, diphtheria, acellular pertussis (Tdap) immunization to prevent infant pertussis. This study's objective was to examine the clinical effectiveness of prenatal Tdap, and whether effectiveness varies by gestational age at immunization.
\end{abstract}

Methods: A nationwide cohort study of pregnant women with deliveries in 2010-2014 and their infants was performed. Commercial insurance claims data were analyzed in 2016-2017 to identify Tdap receipt by the pregnant women, and hospitalizations and outpatient visits for pertussis in their infants until the infants reached 18 months of age. Pertussis occurrence was compared between infants of mothers who received prenatal Tdap (overall and stratified by gestational age at administration) and infants of unvaccinated mothers.

Results: There were 675,167 mother-infant pairs in the cohort. Among infants whose mothers received prenatal Tdap, the rate of pertussis was $43 \%$ lower (hazard ratio $=0.57,95 \% \mathrm{CI}=0.35,0.92$ ) than infants whose mothers did not receive prenatal or postpartum Tdap; this reduction was consistent across pertussis definitions (hazard ratio for inpatient-only pertussis $=0.32,95 \% \mathrm{CI}=0.11$, 0.91). Pertussis rates were also lower for infants whose mothers received Tdap during the third trimester. Infants whose mothers received Tdap at $<27$ weeks of gestation did not experience reductions in pertussis rates (hazard ratio for pertussis $=1.10,95 \% \mathrm{CI}=0.54,2.25$ ).

Conclusions: Infants of mothers who received prenatal Tdap experienced half the rate of pertussis as compared with infants of unimmunized mothers. These results do not provide evidence to support changing the currently recommended timing of Tdap administration in pregnancy.

Am J Prev Med 2018;55(2):159-166.

\section{INTRODUCTION}

$\mathrm{P}$ ertussis, or respiratory infection caused by Bordetella pertussis, has been rising in incidence in the U.S. since $2000 .^{1}$ In 2015 , there were 20,762 pertussis cases reported to the National Notifiable Diseases Surveillance System, with an annual incidence rate of $99 / 100,000$ in infants under age 6 months. ${ }^{1}$ Disease in infants is more likely to be severe and result in hospitalization, ${ }^{2,3}$ apnea, and pneumonia. ${ }^{4,5}$ Most concerning, infants accounted for between $50 \%$ and $92 \%$ of all pertussis-related deaths. ${ }^{1}$

To reduce the burden of pertussis in children, the Centers for Disease Control and Prevention (CDC)
From the ${ }^{1}$ Department of Family Medicine, University of North Carolina at Chapel Hill, Chapel Hill, North Carolina; ${ }^{2}$ Department of Epidemiology, University of North Carolina at Chapel Hill, Chapel Hill, North Carolina; ${ }^{3}$ Division of Infectious Diseases, Department of Medicine, Washington University School of Medicine, St. Louis, Missouri; ${ }^{4}$ Division of Public Health Sciences, Department of Surgery, Washington University School of Medicine, St. Louis, Missouri; ${ }^{5}$ NoviSci, LLC, Durham, North Carolina; ${ }^{6}$ Department of Obstetrics and Gynecology, University of North Carolina at Chapel Hill, Chapel Hill, North Carolina; ${ }^{7}$ Division of Infectious Diseases, Department of Medicine, University of North Carolina at Chapel Hill, Chapel Hill, North Carolina; ${ }^{8}$ Department of Biostatistics, University of North Carolina at Chapel Hill, Chapel Hill, North Carolina; and ${ }^{9}$ RTI Health Solutions, Research Triangle Park, North Carolina

Address correspondence to: Sylvia Becker-Dreps, MD, MPH, UNC Department of Family Medicine, 590 Manning Drive, Chapel Hill NC 27599. E-mail: sbd@unc.edu.

$0749-3797 / \$ 36.00$

https://doi.org/10.1016/j.amepre.2018.04.013 
recommends that all infants receive the primary series of diphtheria, tetanus, acellular pertussis $(\mathrm{DTaP})$ at ages 2 , 4, and 6 months, with booster doses at ages 15-18 months and 4-6 years. However, maximal protection is typically not attained until after the third dose of the vaccine at age 6 months, ${ }^{6,7}$ leaving young infants at higher risk of pertussis. Additional CDC recommendations have been made in recent years, including the provision of a booster dose of tetanus, diphtheria, acellular pertussis (Tdap) to adolescents and Tdap immunization of close adult and adolescent contacts of infants. ${ }^{8,9}$ These strategies have had limited success in decreasing rates of infant pertussis. Therefore, in 2011, the CDC recommended that pregnant women who previously had not received Tdap receive a one-time Tdap booster in pregnancy to provide passive immunity to their infants through transplacental antibody transfer. ${ }^{9}$ New immunogenicity data following the recommendation showed that anti-pertussis antibodies are short-lived and mothers immunized before the current pregnancy or in early pregnancy may have insufficient pertussisspecific antibodies to protect their infants against infection. ${ }^{10}$ Subsequently, the CDC recommended the provision of Tdap in each pregnancy, with the optimal timing of administration at 27-36 weeks of gestation. ${ }^{11}$

The optimal timing of Tdap immunization during pregnancy is currently under dispute. It is unclear if Tdap immunization in the second trimester of pregnancy could result in increased protection as compared with the third trimester, the currently recommended optimal timing. ${ }^{12,13}$ The CDC recommendations for Tdap during pregnancy and its optimal timing were primarily informed by immunologic studies; limited clinical efficacy data exist on the best timing of Tdap administration during pregnancy. Another important concern is whether passively acquired maternal antibodies may blunt the infant's active immune response to the primary series of DTaP, potentially leaving infants at higher risk for pertussis once passively acquired antibodies wane over time. This concern is based on immunologic studies showing that infants whose mothers received Tdap during pregnancy or whose mothers had high titers of anti-pertussis antibodies had weaker antibody responses to pertussis antigens following the primary DTaP series. $^{14,15}$ The clinical significance of these immunologic findings is not known.

The goal of this study is to determine the clinical effectiveness of prenatal Tdap in the prevention of infant pertussis, and to understand if effectiveness varies by gestational age at Tdap administration. Further, the study examines rates of pertussis after the age of 6 months (the recommended age at primary DTaP series completion), to determine whether the immunologic finding of blunting by maternal antibodies has clinical relevance. If findings show that Tdap in pregnancy is highly effective at preventing infant pertussis, this may improve acceptability and uptake of the vaccine in pregnant women.

\section{METHODS}

\section{Data Sample}

An observational cohort of pregnant women and their infants (defined here as age $\leq 18$ months ) delivered between June 2010 and December 2014 was constructed using the Truven Health Analytics MarketScan (B) Commercial Claims and Encounters Databases (copyright( 2016 Truven Health Analytics Inc., all rights reserved), which contains insurance enrollment and billing data for commercially insured employees, spouses, and dependents from $\cong 100$ large employers around the U.S. ${ }^{16}$ These databases include billed, adjudicated, and paid insurance claims for inpatient and outpatient health facility visits, procedures, and their associated diagnosis codes; outpatient claims for medications filled by pharmacies; and claims for performing laboratory tests, but they do not include linkage back to the medical record to access the results of laboratory tests. Mothers with delivery claims (ICD-9-CM codes V27.0-V27.6) were linked within family enrollment groupings to newborns covered on the same insurance plan with birth codes (ICD-9-CM codes V30-V37) occurring within 30 days of the mother's delivery codes (to allow variation in the beginning of infant enrollment). Infants covered under insurance plans different from the mothers' would be unable to match. Enrollment was restricted to the first delivery per woman occurring between June 2010 and December 2014 , and to those with singleton deliveries occurring $>26$ weeks of gestational age. Time periods prior to the recommendation were included to provide a better comparison group of unvaccinated women to avoid solely comparing recommendation-compliant women with noncompliant women after the recommendations were given, which could potentially introduce confounding by access or attitudes to health care. Methods used for estimating gestational age at delivery and vaccination have been described previously. ${ }^{17}$ In brief, the hierarchical algorithm estimated gestational age based on diagnosis and procedure codes assigned to the mother and infant related to pre-maturity, postmaturity, and gestational length. Continuous insurance enrollment was required for mothers from estimated pregnancy onset to 7 days postdelivery to fully characterize maternal characteristics and vaccination status, and for infants until 7 days post-delivery. Infants were followed until censoring at the first occurrence of either the end of follow-up (6 months or 18 months, depending on the analysis) or the end of the study period (December 31, 2014), or disenrollment in the insurance claims database. Clinical and demographic characteristics were collected using diagnosis, procedure, and medication claims and enrollment information. Potential confounding variables, including demographic and clinical characteristics, were identified from the mothers during pregnancy and delivery, and from the infants at delivery and during the first 7 days, prior to the beginning of follow-up.

\section{Measures}

Maternal Tdap immunization was identified through procedure codes (Current Procedural Terminology code 90715, ICD-9-CM 99.37, 99.39) from estimated pregnancy onset until 7 days postdelivery, with the administration timing categorized as prenatal (from pregnancy onset to 2 weeks prior to delivery-also stratified 
$<27$ weeks, or $\geq 27$ weeks to 2 weeks prior to delivery) or postpartum (from 2 weeks prior to delivery to 7 days postdelivery). Mothers vaccinated in the 2 weeks prior to delivery were categorized as postpartum, as it takes approximately 2 weeks for the full immune response to the vaccine; in these cases, the mother would be most similar to mothers who have received Tdap vaccination in the postpartum period.

Infants were followed from day 7 post-delivery up to age 18 months for evidence of pertussis. As laboratory test results are not contained in the claims databases, three different claims-based pertussis definitions were employed, relying on submitted diagnoses, tests ordered, and antibiotics dispensed, as follows:

1. Pertussis, the primary outcome, included either an inpatient pertussis diagnosis alone or an outpatient pertussis diagnosis plus antibiotic treatment with azithromycin, clarithromycin, erythromycin, or trimethoprim/sulfamethoxazole within 7 days. In the outpatient setting, the results of laboratory testing may not be accessible to the treating physician at the time the diagnosis code is assigned on the insurance claim. Therefore, the requirement for an appropriate antibiotic claim was added to outpatient pertussis visit claims to increase the specificity of the outpatient pertussis diagnosis.

2. Inpatient-only pertussis included only inpatient pertussis diagnoses. This is likely the most specific of the three definitions, as diagnosis codes for hospital claims are assigned on hospital discharge; laboratory tests performed during the hospitalization would commonly be completed prior to patient discharge, and therefore, the hospital physician would have this information prior to assigning a diagnosis code for the hospitalization.

3. Possible pertussis includes both definitions above, and also includes outpatient visits with a diagnosis of cough, plus a claim for a pertussis laboratory test, and antibiotic treatment with a macrolide or trimethoprim/sulfamethoxazole within 7 days. This is the broadest of the three definitions and includes the scenario where the provider suspects pertussis in the outpatient setting but may not yet have laboratory results to support the diagnosis.

\section{Statistical Analysis}

Associations of prenatal (overall and stratified by timing) and postpartum maternal immunization with infant pertussis were assessed by estimating hazard ratios (HRs) and 95\% CIs using a multivariable Cox proportional hazards model, using monthly intervals. HRs were adjusted for demographic and clinical characteristics of the mother and infant. Season and cumulative number of infant DTaP doses received were included as monthly time-varying covariates in the Cox model. ${ }^{18}$ Complete case analysis was performed, where infants with missing covariates were excluded from the multivariable analysis. To assess associations of maternal immunization with outcomes both before and after the recommended age for completion of the infant's primary DTaP series, infant follow-up was stratified into two windows: 7 days to 6 months, and 6 to 18 months. Final multivariable models were adjusted for infant's sex, year of delivery, number of infant DTaP doses received (time-varying, monthly), season (timevarying, monthly), residence in a metropolitan statistical area (binary, as a measure of urbanicity), U.S. region, other children covered on insurance plan (categorized as zero, one, two or more), premature birth, stay in the neonatal intensive care unit during the first week of life, and maternal age. A sensitivity analysis limited to infants aged $<2$ months was also performed. Analyses were performed in 2016-2017 using SAS, version 9.4. This study was approved by the IRB of the University of North Carolina at Chapel Hill.

\section{RESULTS}

Among 1,079,034 pregnant women with live-born deliveries who met eligibility criteria between 2010 and 2014, a total of $675,167(62.6 \%)$ were successfully matched to a newborn. These 675,167 infants contributed a total of 672,497 person-years of follow-up time; on average, each infant contributed 355 days ( $\mathrm{SD}=191$ days). Baseline characteristics of mother-infant pairs are shown in Table 1. Characteristics of mothers who were matched to newborns were similar to the overall sample of mothers (Appendix Table 1, available online).

Over the time period studied, 90,445 (13.4\%) women received prenatal Tdap (were vaccinated during pregnancy $>2$ weeks prior to delivery). There were 5,872 $(0.9 \%)$ women vaccinated in the 2 weeks prior to delivery and were included in the postpartum Tdap group, along with $36,470(5.4 \%)$ women who received the vaccine on the day of delivery or in the 7 days after delivery. Among infants whose mothers received prenatal Tdap, the rate of pertussis was $43 \%$ lower $(\mathrm{HR}=0.57,95 \% \mathrm{CI}=0.35,0.92)$, and the rate of inpatient-only pertussis was $68 \%$ lower $(\mathrm{HR}=0.32,95 \% \mathrm{CI}=0.11,0.91)$ than infants whose mothers did not receive prenatal or postpartum Tdap (Table 2). When evaluating prenatal Tdap effectiveness by timing of immunization, infants whose mothers received prenatal Tdap at $\geq 27$ weeks experienced lower rates of pertussis compared with infants of unimmunized mothers $(\mathrm{HR}=0.42,95 \% \mathrm{CI}=0.23,0.78$, for pertussis and $\mathrm{HR}=0.30,95 \% \mathrm{CI}=0.09,0.97$, for inpatient-only pertussis). Infants whose mothers received Tdap at $<27$ weeks did not experience reductions in pertussis rates $(\mathrm{HR}=1.10,95 \% \mathrm{CI}=0.54,2.25)$. Although these infants tended to have lower rates of pertussis hospitalizations and possible pertussis (Table 2), inferences were imprecise because of limited numbers of mothers receiving Tdap at $<27$ weeks. Similarly, infants whose mothers received Tdap in the postpartum period tended to have lower pertussis rates compared with infants whose mothers did not receive Tdap, but inferences were imprecise.

The majority of pertussis cases occurred in the first 6 months of life (Table 3). During this time, infants whose mothers received prenatal Tdap had a $46 \%$ lower rate of pertussis $(\mathrm{HR}=0.54,95 \% \mathrm{CI}=0.31,0.94)$, and a $75 \%$ lower rate of inpatient-only pertussis $(\mathrm{HR}=0.25,95 \%$ 
Table 1. Characteristics of Mother-Infant Pairs by Receipt of Maternal Tdap

\begin{tabular}{|c|c|c|c|c|c|}
\hline Characteristic & $\begin{array}{c}\text { Total, } n(\%) \\
(\mathrm{N}=675,167)\end{array}$ & $\begin{array}{c}<27 \text { weeks } \\
\text { prenatal, } n(\%) \\
(n=16,273)\end{array}$ & $\begin{array}{c}\geq 27 \text { weeks } \\
\text { prenatal, } n(\%) \\
(n=74,172)\end{array}$ & $\begin{array}{c}\text { Postpartum, }^{\mathrm{b}} \\
n(\%) \\
(n=42,342)\end{array}$ & $\begin{array}{c}\text { Unvaccinated, }^{\mathrm{c}} \\
n(\%) \\
(n=542,380)\end{array}$ \\
\hline $\begin{array}{l}\text { Maternal age, mean } \\
\text { (SD) }\end{array}$ & $30.3(4.6)$ & $30.4(4.5)$ & $30.5(4.4)$ & $30.1(4.4)$ & $30.2(4.6)$ \\
\hline \multicolumn{6}{|l|}{ Infant sex } \\
\hline Male & $344,010(51.0)$ & $8,225(50.5)$ & $37,603(50.7)$ & 21,583 (51.0) & 276,599 (51.0) \\
\hline Female & 331,157 (49.1) & $8,048(49.5)$ & 36,569 (49.3) & 20,759 (49.0) & 265,781 (49.0) \\
\hline \multicolumn{6}{|l|}{ Birth year } \\
\hline 2010 & $112,093(16.6)$ & $225(1.4)$ & $398(0.5)$ & 3,503 (8.3) & 107,967 (19.9) \\
\hline 2011 & $152,179(22.5)$ & $1,402(8.6)$ & $1,132(1.5)$ & 9,968 (23.5) & $139,677(25.8)$ \\
\hline 2012 & $161,932(24.0)$ & 3,117 (19.2) & $6,894(9.3)$ & $12,290(29.0)$ & $139,631(25.7)$ \\
\hline 2013 & 115,563 (17.1) & $5,011(30.8)$ & 20,685 (27.9) & 8,057 (19.0) & $81,810(15.1)$ \\
\hline 2014 & $133,400(19.8)$ & $6,518(40.1)$ & $45,063(60.8)$ & $8,524(20.1)$ & 73,295 (13.5) \\
\hline \multicolumn{6}{|l|}{ U.S. region ${ }^{d}$} \\
\hline Northeast & $110,444(16.4)$ & 2,403 (14.8) & $11,293(15.2)$ & 5,863 (13.9) & 90,885 (16.8) \\
\hline Midwest & $179,380(26.6)$ & 4,269 (26.2) & 21,696 (29.3) & $10,321(24.4)$ & $143,094(26.4)$ \\
\hline South & $241,110(35.7)$ & 4,482 (27.5) & 21,544 (29.1) & $16,388(38.7)$ & 198,696 (36.6) \\
\hline West & $130,787(19.4)$ & $4,664(28.7)$ & $17,522(23.6)$ & 8,882 (21.0) & $99,719(18.4)$ \\
\hline Lives in MSA & $586,911(86.9)$ & $14,419(88.6)$ & $65,771(88.7)$ & 36,965 (87.2) & 469,789 (86.6) \\
\hline $\begin{array}{l}\text { Received obstetric } \\
\text { blood panel }^{e}\end{array}$ & $382,611(56.7)$ & 9,347 (57.4) & $42,134(56.8)$ & 26,507 (62.6) & 304,623 (56.2) \\
\hline Received ultrasound ${ }^{e}$ & $551,774(81.7)$ & $15,014(92.3)$ & $66,612(89.8)$ & $36,147(85.4)$ & 434,001 (80.0) \\
\hline $\begin{array}{l}\text { Received influenza } \\
\text { vaccine }\end{array}$ & $158,179(23.4)$ & $8,468(52.0)$ & $36,162(48.8)$ & $10,735(25.4)$ & 102,814 (19.0) \\
\hline $\begin{array}{l}\text { Hospitalizations, } \\
\text { mean (SD) }\end{array}$ & $0.008(0.098)$ & $0.006(0.092)$ & $0.005(0.081)$ & $0.008(0.100)$ & $0.008(0.101)$ \\
\hline $\begin{array}{l}\text { Maternal } \\
\text { hypertension }\end{array}$ & $92,897(13.8)$ & 2,279 (14.0) & 10,315 (13.9) & 5,949 (14.1) & $74,354(13.7)$ \\
\hline Gestational diabetes & $99,473(14.7)$ & 2,404 (14.8) & $11,358(15.3)$ & 6,485 (15.3) & $79,226(14.6)$ \\
\hline Preterm delivery & $50,338(7.5)$ & 1,697 (10.4) & $3,530(4.8)$ & $3,498(8.3)$ & $41,613(7.7)$ \\
\hline $\begin{array}{l}\text { Delivered by } \\
\text { cesarean section }\end{array}$ & 229,157 (33.9) & $5,445(33.5)$ & 23,143 (31.2) & $14,468(34.2)$ & 186,101 (34.3) \\
\hline $\begin{array}{l}\text { Other covered } \\
\text { children on plan, } \\
\text { mean (SD) }\end{array}$ & $1.9(1.0)$ & $1.7(0.9)$ & $1.7(0.9)$ & $1.8(1.0)$ & $1.9(1.0)$ \\
\hline $\begin{array}{l}\text { Received all DTaP on } \\
\text { time }^{f}\end{array}$ & $496,789(73.6)$ & 13,077 (80.4) & $62,479(84.2)$ & $32,891(77.7)$ & $388,342(71.6)$ \\
\hline $\begin{array}{l}\text { Total follow-up in } \\
\text { study, person-years }\end{array}$ & 672,497 & 14,122 & 52,364 & 39,840 & 566,172 \\
\hline
\end{tabular}

'Vaccinated after 27 weeks' gestational age but prior to 2 weeks before delivery.

${ }^{b}$ Vaccinated in the 2 weeks prior to delivery, on the day of delivery, or in the 7 days after delivery.

${ }^{c}$ Unvaccinated during pregnancy or in the postpartum period.

${ }^{\mathrm{d}}$ Percentages do not add up to $100 \%$ because of missing data.

${ }^{\mathrm{e}}$ Assessed from pregnancy onset to 20 weeks of gestation.

fOn-time defined as not more than 1 month late for any DTaP vaccine, according to Centers for Disease Control and Prevention (CDC) recommended schedule, by the end of the follow-up period.

DTaP, diphtheria, tetanus, acellular pertussis immunization; MSA, metropolitan statistical area or group of counties containing at least one urbanized area that has a population of at least 50,000; Tdap, tetanus, diphtheria, acellular pertussis immunization.

$\mathrm{CI}=0.08,0.80)$, as compared with infants whose mothers did not receive prenatal or postpartum Tdap. A sensitivity analysis limited to infants aged $<2$ months showed similar HR estimates as for those aged $<6$ months, but because of the smaller number of cases, estimates for those aged $<2$ months had wider CIs (Appendix Table 2, available online). Between 6 and 18 months of life, there were no differences in pertussis rates by receipt of prenatal Tdap after adjustment for the infant's DTaP receipt $(\mathrm{HR}=0.69,95 \% \mathrm{CI}=0.26,1.86$, for pertussis and $\mathrm{HR}=2.60,95 \% \quad \mathrm{CI}=0.15,46.2$, for inpatient-only pertussis). 
Table 2. Rates of Pertussis up to Age 18 months by Receipt and Timing of Maternal Tdap

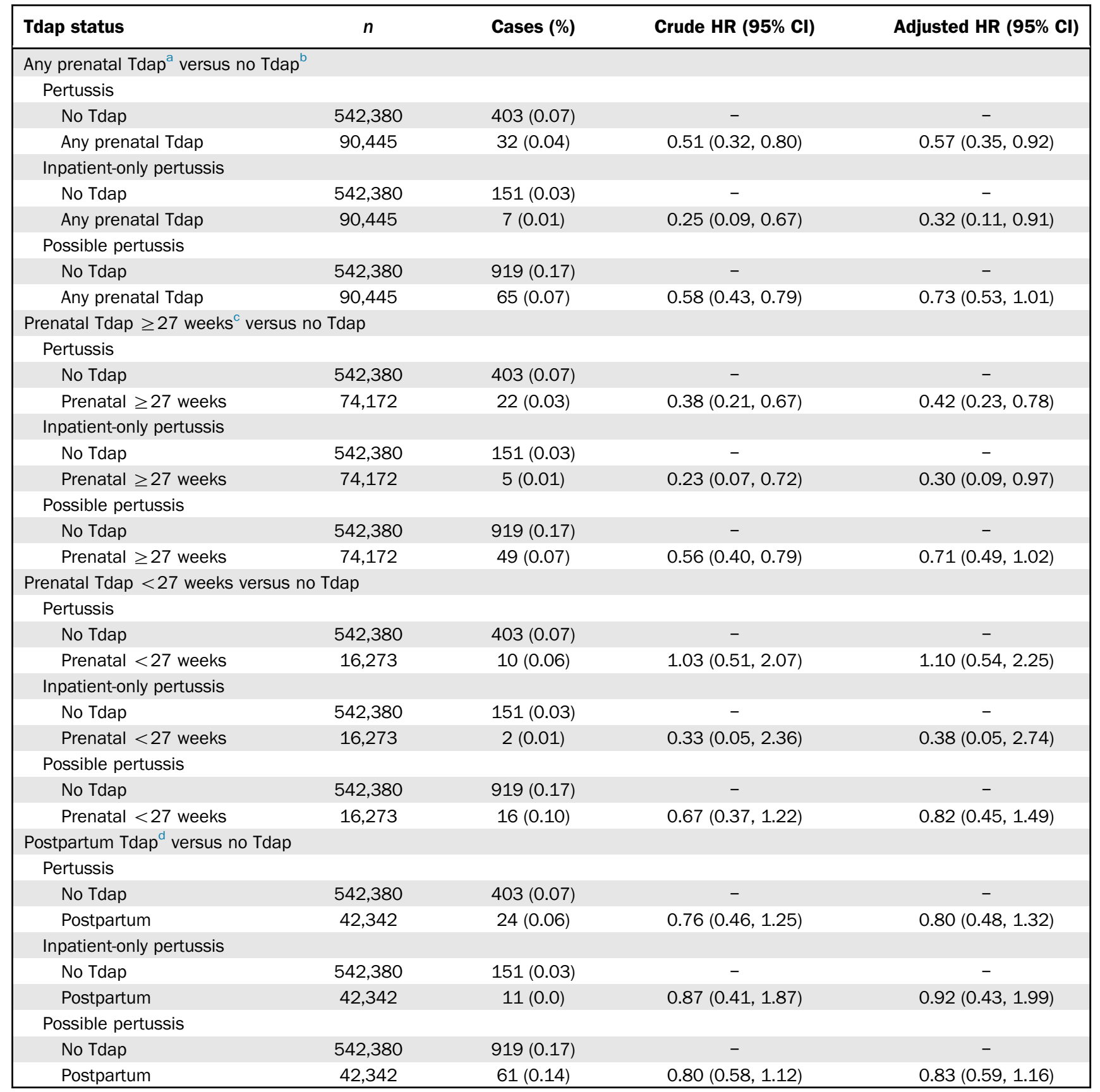

Note: Models were adjusted for infant's sex; year of delivery; number of infant diphtheria, tetanus, acellular pertussis (DTaP) doses received (timevarying, monthly); season (time-varying, monthly); residence in a metropolitan statistical area (binary, as a measure of urbanicity); U.S. region; other children covered on insurance plan (categorized as $0,1, \geq 2$ ); premature birth; stay in the neonatal intensive care unit during the first week of life; maternal age.

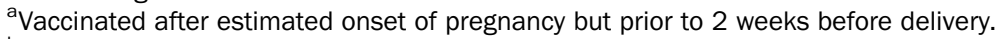

${ }^{b}$ Unvaccinated during pregnancy or in the postpartum period.

'Vaccinated after 27 weeks' gestational age but prior to 2 weeks before delivery.

dVaccinated in the 2 weeks prior to delivery, on the day of delivery, or in the 7 days after delivery.

$\mathrm{HR}$, hazard ratio; Tdap, tetanus-diphtheria-acellular pertussis immunization.

\section{DISCUSSION}

This study compared clinical outcomes of 675,167 privately insured U.S. infants by maternal Tdap immunization status. Infants whose mothers received Tdap immunization during pregnancy experienced about half the rate of pertussis as compared with infants whose 
Table 3. Rates of Pertussis by Receipt of Prenatal Tdap, Stratified by Infant Age

\begin{tabular}{|c|c|c|c|c|}
\hline Tdap status & $n$ & Cases(\%) & Crude HR (95\% Cl) & Adjusted HR (95\% CI) \\
\hline \multicolumn{5}{|l|}{ Pertussis } \\
\hline Any prenatal ${ }^{\mathrm{b}}$ & 90,445 & $22(0.03)$ & $0.45(0.27,0.76)$ & $0.54(0.31,0.94)$ \\
\hline \multicolumn{5}{|c|}{ Inpatient-only pertussis } \\
\hline \multicolumn{5}{|c|}{ Possible pertussis } \\
\hline No Tdap & 542,380 & $419(0.08)$ & - & - \\
\hline Any prenatal & 90,445 & $30(0.03)$ & $0.51(0.34,0.79)$ & $0.65(0.41,1.02)$ \\
\hline \multicolumn{5}{|c|}{ Age 6 months to 18 months } \\
\hline \multicolumn{5}{|l|}{ Pertussis } \\
\hline No Tdap & 422,214 & $6(0.00)$ & - & - \\
\hline Any prenatal & 53,508 & $1(0.00)$ & $2.97(0.31,28.6)$ & $2.60(0.15,46.2)$ \\
\hline \multicolumn{5}{|c|}{ Possible pertussis } \\
\hline No Tdap & 422,214 & $468(0.11)$ & - & - \\
\hline Any prenatal & 53,508 & $35(0.07)$ & $0.70(0.46,1.08)$ & $0.86(0.54,1.37)$ \\
\hline
\end{tabular}

Note: Models were adjusted for infant's sex; year of delivery; number of infant diphtheria, tetanus, acellular pertussis (DTaP) doses received (timevarying, monthly); season (time-varying, monthly); residence in a metropolitan statistical area (binary, as a measure of urbanicity); U.S. region; other children covered on insurance plan (categorized as $0,1, \geq 2$ ); premature birth; stay in the neonatal intensive care unit during the first week of life; maternal age.

anvaccinated during pregnancy or in the postpartum period.

${ }^{b}$ Vaccinated after estimated onset of pregnancy but prior to 2 weeks before delivery.

$\mathrm{HR}$, hazard ratio; Tdap, tetanus-diphtheria-acellular pertussis immunization.

mothers did not receive it during pregnancy or in the postpartum period. This study adds to two prior studies from the United Kingdom that showed high effectiveness (approximately 90\%) of prenatal Tdap in infants under age 3 months. ${ }^{19,20}$ A study from six U.S. Emerging Infection Program Network states reported an effectiveness of $78 \%$ during the first 2 months of life, ${ }^{21}$ whereas another study in the Kaiser Permanente population in California reported an effectiveness of $69 \%$ during the first year of life. ${ }^{22}$ The estimates of the benefit of prenatal Tdap in this study, which followed infants up to age 18 months, were weaker than in these previous studies, which examined infants over shorter time intervals. These differences may be because of duration of protection afforded by maternal Tdap immunization. The halflife of transplacental anti-pertussis antibodies is approximately 7 weeks $^{23}$; therefore, the benefits of maternal immunization diminish over time. A sensitivity analysis did not show greater protection of prenatal Tdap for infants during the first 2 months of life as compared with the first 6 months of life, but these estimates became imprecise.

In the analyses of timing of vaccination, infants whose mothers received prenatal Tdap during the third trimester experienced reductions in pertussis cases and hospitalizations as compared with infants of unimmunized mothers. The study did not find statistically significant reductions of infant pertussis rates in mothers who were immunized before the third trimester or in the postpartum period. Therefore, these data do not provide evidence to support a change in the current recommendation for optimal timing of immunization. There may be logistic reasons to recommend Tdap vaccination earlier in pregnancy, such as to provide protection to infants born prematurely; future studies should examine the overall risks and benefits to the population of such a strategy. Further, there was no evidence for an increase in pertussis rates after the typical age of $\mathrm{DTaP}$ primary series completion for infants of immunized mothers compared with infants of unimmunized mothers, to provide clinical relevance to the finding that maternal antibodies may interfere with the infant's response to the primary DTaP series. ${ }^{14,15}$ However, overall numbers of pertussis cases in older infants used in these comparisons were small.

\section{Limitations}

There are limitations of claims-based analyses, including the possibility of misclassification. Three definitions of pertussis were employed. Although inpatient diagnoses may be the 
most specific, as discharge diagnoses are likely made after the results of microbiology laboratory tests are available, many pertussis cases are assessed and treated in the outpatient setting, where diagnosis coding may be based on symptoms rather than confirmed diagnoses, as laboratory results are likely not available during the outpatient visit. Using combinations of pertussis diagnosis codes, more general symptom codes, laboratory testing, and antibiotic treatment coding, three levels of pertussis were defined from confirmed inpatient cases to potential cases. Analyses were performed using all three definitions, and the conclusions were consistent across the pertussis definitions.

Also, although insurance plan enrollment is grouped by families, there is not a direct link identifying the resulting offspring from a woman's delivery within the insurance claims. Mother-infant matchings were based on family grouping and delivery/birth date, but there remains the potential for matching errors, potentially resulting in misclassification of maternal Tdap exposure in newborns. It is unlikely that matching errors would differ by Tdap status, and any resulting nondifferential misclassification would likely bias the results towards the null, thus not explaining the observed protective associations.

In addition, estimates for early immunization were imprecise as the majority of women received the vaccine after 27 weeks of gestational age. Finally, this study was restricted to a privately insured population and may not be generalizable to publicly or uninsured populations. The strengths of the study include a large sample size from a variety of healthcare settings throughout the U.S., and accurate assessment of exposure, as insurance claims are audited and serve as the basis for reimbursement. ${ }^{24}$

\section{CONCLUSIONS}

Prenatal Tdap provided a substantial reduction in infant pertussis during the period of life with the highest pertussis burden. Increasing use of Tdap in pregnancy would be anticipated to reverse the current trends of pertussis incidence among infants in the U.S.

\section{ACKNOWLEDGMENTS}

This work was supported by the National Institute of Allergy and Infectious Diseases (5R21Al115205). The database infrastructure used for this project was funded by the Department of Epidemiology, University of North Carolina (UNC) Gillings School of Global Public Health; the Cecil G. Sheps Center for Health Services Research, UNC; the Comparative Effectiveness Research Strategic Initiative of UNC's Clinical \& Translational Science Award (UL1TR001111); and the UNC School of Medicine. Dr. Becker-Dreps is supported by Grant 2015213 from the Doris Duke Charitable Foundation. The study sponsors had no role in the study design; data collection, analysis, and interpretation; manuscript preparation; or the decision to submit the report for publication. This study was approved by the IRB of the UNC at Chapel Hill (FWA No.: 4801, Study No.: 13-3679). Dr. Butler performed this work when she was at the UNC at Chapel Hill.

Author tasks were as follows: conception of the study: SBD, LM, KAB, DJW, and MGH; data analysis: SBD, AMB, DL, MGH, and JBL; interpretation: SBD, AMB, LJM, KAB, DJW, MGH, and JBL; completed first draft of manuscript: SBD, AMB, and JBL; and reviewed, revised for intellectual content, and approved manuscript: all authors.

Dr. Becker-Dreps received investigator-initiated research funds from Pfizer for a study on pneumococcal vaccines and served as a vaccine consultant (Pfizer). Dr. Butler received investigator-initiated research funds from Amgen, Inc. Dr. Weber served on speakers' bureaus and as a vaccine consultant (Merck, Pfizer). Dr. Layton is a current employee of RTI International, an independent research organization that does contract work for governmental agencies and pharmaceutical companies; he is formerly an employee of UNC, where he received unrestricted salary support from the UNC Center for Pharmacoepidemiology, whose current members include GlaxoSmithKline, Merck, and UCB Biosciences. Drs. McGrath, Boggess, and Hudgens and Ms. Li have no financial disclosures.

\section{SUPPLEMENTAL MATERIAL}

Supplemental materials associated with this article can be found in the online version at https://doi.org/10.1016/j. amepre.2018.04.013.

\section{REFERENCES}

1. Centers for Disease Control and Prevention (CDC). Pertussis: surveillance and reporting, 2012-2016. www.cdc.gov/pertussis/surv-report ing.html. Accessed August 23, 2017.

2. Cortese MM, Baughman AL, Zhang R, et al. Pertussis hospitalizations among infants in the United States, 1993 to 2004. Pediatrics. 2008;121 (3):484-492. https://doi.org/10.1542/peds.2007-1393.

3. Miller CL, Fletcher WB. Severity of notified whooping cough. Br Med J. 1976;1(6002):117-119. https://doi.org/10.1136/bmj.1.6002.117.

4. Eidlitz-Markus T, Mimouni M, Zeharia A. Pertussis symptoms in adolescents and children versus infants: the influence of vaccination and age. Clin Pediatr (Phila). 2007;46(8):718-723. https://doi.org/ 10.1177/0009922807302093.

5. Gordon M, Davies HD, Gold R. Clinical and microbiologic features of children presenting with pertussis to a Canadian pediatric hospital during an eleven-year period. Pediatr Infect Dis J. 1994;13(7):617-622. https://doi.org/10.1097/00006454-199407000-00007.

6. Auerbach BS, Lake AM, Wilson ME, et al. Dose-response to a twocomponent acellular pertussis vaccine in infants and comparison with whole-cell vaccine. Biologicals. 1998;26(2):145-153. https://doi.org/ 10.1006/biol.1998.9999.

7. Bisgard KM, Rhodes P, Connelly BL, et al. Pertussis vaccine effectiveness among children 6 to 59 months of age in the United States, 1998-2001. Pediatrics. 2005;116(2):e285-e294. https://doi.org/10.1542/peds.2004-2759.

8. Broder KR, Cortese MM, Iskander JK, et al. Preventing tetanus, diphtheria, and pertussis among adolescents: use of tetanus toxoid, reduced diphtheria toxoid and acellular pertussis vaccines 
recommendations of the Advisory Committee on Immunization Practices (ACIP). MMWR Recomm Rep. 2006;55(RR-3):1-34.

9. Centers for Disease Control and Prevention (CDC). Updated recommendations for use of tetanus toxoid, reduced diphtheria toxoid and acellular pertussis vaccine (Tdap) in pregnant women and persons who have or anticipate having close contact with an infant aged 12 months - Advisory Committee on Immunization Practices (ACIP), 2011. MMWR Morb Mortal Wkly Rep. 2011;60(41):1424-1426.

10. Healy CM, Rench MA, Baker CJ. Importance of timing of maternal combined tetanus, diphtheria, and acellular pertussis (Tdap) immunization and protection of young infants. Clin Infect Dis. 2013;56(4):539544. https://doi.org/10.1093/cid/cis923.

11. Centers for Disease Control and Prevention (CDC). Updated recommendations for use of tetanus toxoid, reduced diphtheria toxoid, and acellular pertussis vaccine (Tdap) in pregnant women-Advisory Committee on Immunization Practices (ACIP), 2012. MMWR Morb Mortal Wkly Rep. 2013;62(7):131-135.

12. Eberhardt CS, Blanchard-Rohner G, Lemaitre B, et al. Maternal immunization earlier in pregnancy maximizes antibody transfer and expected infant seropositivity against pertussis. Clin Infect Dis. 2016;62 (7):829-836. https://doi.org/10.1093/cid/ciw027.

13. Abu Raya B, Srugo I, Kessel A, et al. The effect of timing of maternal tetanus, diphtheria, and acellular pertussis (Tdap) immunization during pregnancy on newborn pertussis antibody levels-a prospective study. Vaccine. 2014;32 (44):5787-5793. https://doi.org/10.1016/j.vaccine.2014.08.038.

14. Hardy-Fairbanks AJ, Pan SJ, Decker MD, et al. Immune responses in infants whose mothers received Tdap vaccine during pregnancy. Pediatr Infect Dis J. 2013;32(11):1257-1260. https://doi.org/10.1097/ INF.0b013e3182a09b6a.

15. Voysey M, Kelly DF, Fanshawe TR, et al. The influence of maternally derived antibody and infant age at vaccination on infant vaccine responses: an individual participant meta-analysis. JAMA Pediatr. 2017;171(7):637-646. https://doi.org/10.1001/jamapediatrics.2017.0638.
16. MarketScan Database. MarketScan User Guide, Commercial Claims and Encounters, Medicare Supplemental and Coordination of Benefits. Ann Arbor, MI: Thomson Reuters (Healthcare) Inc.; 2008.

17. Butler AM, Layton JB, Li D, et al. Predictors of low uptake of prenatal tetanus toxoid, reduced diphtheria toxoid, and acellular pertussis immunization in privately insured women in the United States. Obstet Gynecol. 2017;129(4):629-637. https://doi.org/10.1097/AOG.0000000000001927.

18. Powell TM, Bagnell ME, editors. Your "survival" guide to using timedependent covariates. SAS Global Forum 2012. http://support.sas.com/ resources/papers/proceedings12/168-2012.pdf. Accessed August 29, 2017.

19. Amirthalingam G, Andrews N, Campbell H, et al. Effectiveness of maternal pertussis vaccination in England: an observational study. Lancet. 2014;384(9953):1521-1528. https://doi.org/10.1016/ S0140-6736(14)60686-3.

20. Dabrera G, Amirthalingam G, Andrews N, et al. A case-control study to estimate the effectiveness of maternal pertussis vaccination in protecting newborn infants in England and Wales, 2012-2013. Clin Infect Dis. 2015;60(3):333-337. https://doi.org/10.1093/cid/ciu821.

21. Skoff TH, Blain AE, Watt J, et al. Impact of the U.S. maternal tetanus, diphtheria, and acellular pertussis vaccination program on preventing pertussis in infants 2 months of age: a case-control evaluation. Clin Infect Dis. 2017;65(12):1977-1983. https://doi.org/10.1093/cid/cix724.

22. Baxter R, Bartlett J, Fireman B, et al. Effectiveness of vaccination during pregnancy to prevent infant pertussis. Pediatrics. 2017;139(5). https://doi.org/10.1542/peds.2016-4091.

23. Vilajeliu A, Ferrer L, Munros J, et al. Pertussis vaccination during pregnancy: Antibody persistence in infants. Vaccine. 2016;34(33): 3719-3722. https://doi.org/10.1016/j.vaccine.2016.05.051.

24. Schneeweiss S, Avorn J. A review of uses of health care utilization databases for epidemiologic research on therapeutics. J Clin Epidemiol. 2005;58(4):323-337. https://doi.org/10.1016/j.jclinepi.2004. 10.012 . 